\title{
Dimensionality reduction in conic section function neural network
}

\section{TULAY YILDIRIM and LALE OZYILMAZ}

Yildiz Technical University, Electronics and Communications Engineering Department, 34349 Besiktas-Istanbul, Turkey

e-mail: \{tulay, ozyilmaz\}@yildiz.edu.tr

MS received 26 November 2001; revised 25 September 2002

\begin{abstract}
This paper details how dimensionality can be reduced in conic section function neural networks (CSFNN). This is particularly important for hardware implementation of networks. One of the main problems to be solved when considering the hardware design is the high connectivity requirement. If the effect that each of the network inputs has on the network output after training a neural network is known, then some inputs can be removed from the network. Consequently, the dimensionality of the network, and hence, the connectivity and the training time can be reduced. Sensitivity analysis, which extracts the cause and effect relationship between the inputs and outputs of the network, has been proposed as a method to achieve this and is investigated for Iris plant, thyroid disease and ionosphere databases. Simulations demonstrate the validity of the method used.
\end{abstract}

Keywords. Conic section function neural network; dimensionality reduction; hardware implementation; sensitivity analysis.

\section{Introduction}

Many analog, digital, and mixed hardware implementations have been presented in the recent literature (Ramacher \& Rückert 1991; Delgado-Frias \& Moore 1994; Elmasry 1994). One of the main problems to be solved when considering the hardware design is the high connectivity requirement. In order to reduce the connectivity, various statistical solutions have been used in recent years, such as principal component analysis, Karhunen-Loeve transform, discrete stochastic complexity criterion etc. (Fakhr et al 1994; Ham \& Kostanic 2001). In this paper, sensitivity analysis has been proposed as an alternative to statistical methods for conic section function neural network (CSFNN). The purpose of choosing this specific network is that it includes both multilayer perceptron (MLP) and radial basis functions (RBF), which are wellknown feedforward neural network structures.

MLP is the most common neural network model, consisting of successive linear transformations followed by processing with nonlinear activation functions. It represents a generalisation of the single layer perceptron, which is only capable of constructing linear decision boundaries and simple logic functions. However, by cascading perceptrons in layers complex decision boundaries and arbitrary Boolean expressions can be implemented (Haykin 1994). 
MLP network consists of a set of source nodes that constitute the input layer, one or more hidden layers of computation nodes, and an output layer. Each layer computes the activation function of a weighted sum of the layer's inputs. The input signal propagates through the network in a forward direction, on a layer-by-layer basis. The learning algorithm for MLPs can be expressed using generalised delta rule and gradient descent since they have non-linear activation functions.

In general form of an MLP network, the $x_{i}$ inputs are fed into the first layer of $x_{h, 1}$ hidden units. In the input units, no processing takes place. The activation of a hidden unit (neuron $j$ ) is a function $f_{j}$ of the weighted inputs plus a bias, as below,

$$
x_{p j}=f_{j}\left(\sum_{i} w_{j i} x_{p i}+\theta_{j}\right)=f_{j}\left(y_{p j}\right),
$$

where $w_{j i}$ is the weight of input $i$ to neuron $j, x_{p i}$ is input $i$, that is, output $i$ from the previous layer, for input pattern $p$ and $\theta_{j}$ is the threshold value. The output of the hidden units is distributed over the next layer of $x_{h, 2}$ hidden units until the last layer of hidden units, of which the outputs are fed into a layer of $x_{o}$ output units. This structure can partition the pattern space using hyperplanes.

The RBF approach consists of modelling an input-output mapping as a linear combination of radially symmetric functions (Broomhead \& Lowe 1988; Poggio \& Girosi 1990). The properties of RBF's are attracting a great deal of interest due to their rapid training, generality, and simplicity. In the context of an RBF neural network, the hidden units provide a set of "kernel functions" that constitute an arbitrary "basis" for the input patterns when they are expanded into the hidden-unit space; these kernel functions are called radial basis functions. These are generally non-linear functions that are built up into one function that can partition the pattern space successfully using hyperspheroids. Each kernel is associated with an activation region from the input space with respect to the data sample local densities and its output is fed to an output unit (Haykin 1994).

An RBF network can be considered as a two-layer fully interconnected feedforward network whose output nodes form a linear combination of the basis functions computed by the hidden layer nodes. The network is designed to perform a non-linear mapping from the input space to hidden space, followed by a linear mapping from the hidden space to the output space. An $\mathrm{RBF}$ is a multidimensional function which depends on the distance $r=\|x-c\|$ (where $\|\cdot\|$ denotes a vector form) between the input vector $x$ and the centre $c$. The first layer of an RBF network computes this distance of the input to the network to a set of stored memories. Each basis function is a nonlinear function of a corresponding distance. The basis functions in the hidden layer produce a localised response to the input vector. The basis functions encode the inputs by computing how close the inputs are to the centres of the receptive field instead of just evaluating the weighted sum of the inputs. The general form of an RBF is

$$
F(x)=\sum_{i=1}^{N} w_{i} \phi\left(\left\|x-c_{i}\right\|\right),
$$

where $w_{i}$ are the coefficients or weights of $c_{i}, N$ is the number of centres. The known data points $c_{i}$ is taken to be the centres of radial basis functions. $x$ is the input to the network. $\left\{\phi\left(\left\|x-c_{i}\right\|\right) \mid i=1,2, \ldots, N\right\}$ is a set of $N$ arbitrary functions. This functional form is preselected with the centres $c_{i}$ being some fixed points in $N$-dimensional space appropriately spanning the input domain. 
The CSFNN used in this work (Dorffner 1994; Yildirim \& Marsland 1997) is a hybrid network based on hyperplanar and hyperspherical decision regions as special cases of conic sections. These are decision regions of MLP and RBF networks respectively. The idea is to generalise unit function that contains all decision regions by providing a relationship between RBF and MLP units. The activation for CSFNN is derived using analytical equations for a cone. This is given by

$$
y_{p j}^{(1)}=\sum_{i=1}^{m}\left(x_{p i}-c_{i j}\right) w_{i j}-\cos \omega_{j}\left(\sum_{i=1}^{m}\left(x_{p i}-c_{i j}\right)^{2}\right)^{1 / 2},
$$

where $w_{i j}$ refers to the weights between the input and hidden layer units in an MLP network, $c_{i j}$ refers to the centre coordinates in an RBF network, and $p$ number of patterns. This equation consists of two major parts analogous to the MLP and RBF networks. $w_{i j}$ refers to the weights for MLP network and $c_{i j}$ refers to the centre coordinates in an RBF network. $\omega_{j}$ is the opening angle of a cone to provide transition from radial basis function network to multilayer perceptron. Hence, (3) simply turns into the propagation rule of an MLP network, which is the dot product when the $\omega$ is $\pi / 2$. Second part of the equation gives the Euclidean distance between the inputs and the centres for an RBF network when the $\omega$ is $\pi / 4$ (Dorffner 1994). The general structure of CSFNN is shown in figure 1.

The learning algorithm for CSFNN involves the determination of the centres and updating weights, centres, and the vertex angle of cone, $\omega$, appropriately. The centres are determined using orthogonal least squares learning algorithm (Chen et al 1991). The vertex angle is chosen in such a way that the network would start as RBF. CSFNN shows an MLP characteristic at the output layer while it shows both RBF and MLP characteristics in the hidden layer. The weights, centres and angle values are updated using error back propagation so that the network would converge quickly (Yildirim \& Marsland 1997; Ozyilmaz 2000).

In this paper, to reduce the dimensionality of this hybrid network, sensitivity analysis has been used as a method. This method is explained in the following section. Then, using thyroid

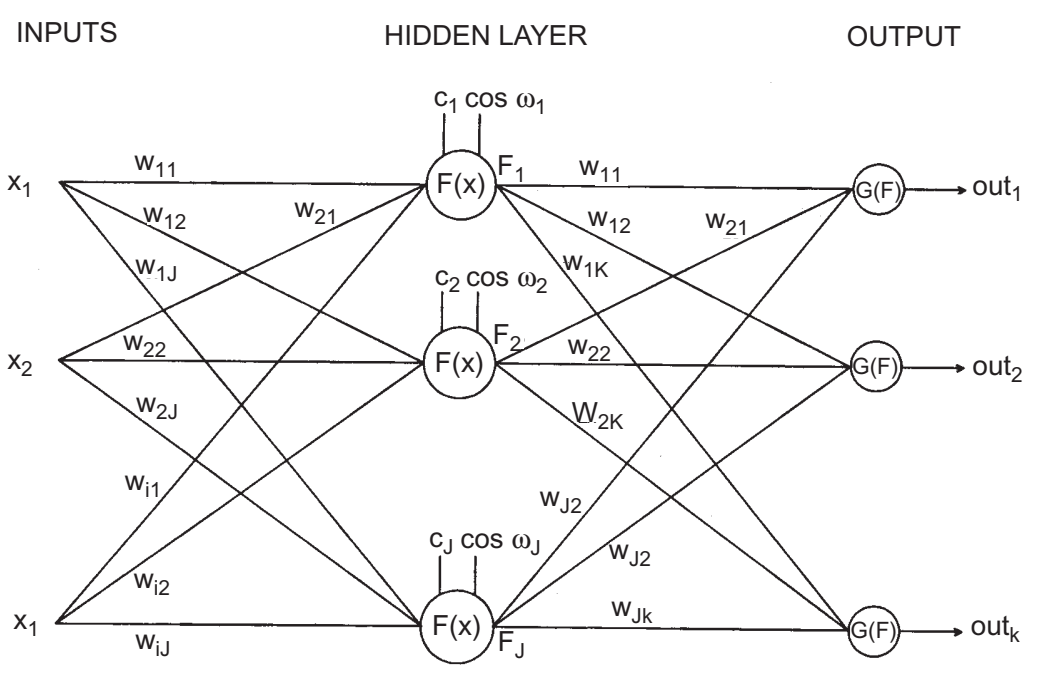

Figure 1. Conic section function neural network structure. 
disease, Iris plant pattern recognition and ionosphere databases, the proposed method has been investigated and the results given in the last section.

\section{Method for dimensionality reduction of conic section function neural network}

Dimensionality reduction in CSFNN is performed by sensitivity analysis. This allows determining the effect on the network outputs due to the changes in the inputs of network. Once it is achieved, inputs with a small sensitivity can be removed from the network after training the network. Hence, the dimensionality of network, consequently, the connectivity and the training time can be reduced.

The unnormalised sensitivity function $S[y, x]$ given in (4) is defined to find the changes in the system output $y$ depending on the change in the system input $x$ (Acar 1979; Basturk \& Gurgen 1993).

$$
\Delta y=S[y, x] \Delta x \Rightarrow S[y, x]=\partial y / \partial x .
$$

If any of the input channels to the network changes slightly, a corresponding change in the ouput(s) is observed after training of the network. For sensitivity analysis, it is necessary to determine how the changes in the $i$ th input of the input layer for pattern $p, x_{p i}$, affects the $k$ th output of output layer; that is, how the $\partial$ out $_{p k}^{(2)} / \partial x_{p i}$ value needs to be computed (Ozyilmaz 2000; Ozyilmaz \& Yildirim 2000). According to this, the sensitivity analysis formulas for conic section function neural network are obtained as follows.

$$
\begin{aligned}
\operatorname{out}_{p k}^{(2)} & =1 /\left(1+e^{-y_{p k}^{(2)}}\right), \\
y_{p k}^{(2)} & =\sum_{j=1}^{n} w_{k j} \text { out }_{p j}^{(1)} .
\end{aligned}
$$

Here, the contribution of the RBF part in (3) is eliminated in (6) as the CSFNN output shows an MLP characteristic. Using the chain rule, the derivative can be written as

$$
\frac{\partial \text { out }_{p k}^{(2)}}{\partial x_{p i}}=\frac{\partial \text { out }_{p k}^{(2)}}{\partial y_{p k}^{(2)}} \cdot \frac{\partial y_{p k}^{(2)}}{\partial x_{p i}} .
$$

The first term of the product in (7) is the derivative of logistic function given by (5).

$$
\frac{\partial \text { out }_{p k}^{(2)}}{\partial y_{p k}^{(2)}}=\text { out }_{p k}^{(2)}\left(1-\text { out }_{p k}^{(2)}\right) .
$$

Equation (7) can be rewritten as

$$
\frac{\partial \text { out }_{p k}^{(2)}}{\partial x_{p i}}=\frac{\partial y_{p k}^{(2)}}{\partial x_{p i}} \operatorname{out}_{p k}^{(2)}\left(1-\operatorname{out}_{p k}^{(2)}\right) .
$$

The second term of the product in (7) is

$$
\frac{\partial y_{p k}^{(2)}}{\partial x_{p i}}=\sum_{j=1}^{n} w_{k j} \frac{\partial \operatorname{out}_{p j}^{(1)}}{\partial x_{p i}} .
$$


Here, to obtain the derivative at the right side of the formula, the contributions of both RBF and MLP parts in (3) are taken into account, as the output of the hidden layer in CSFNN shows the characteristics of both RBF and MLP networks.

As a result, using the equations above, the formula of sensitivity analysis for CSFNN is obtained as

$$
\frac{\partial \text { out }_{p k}^{(2)}}{\partial x_{p i}}=\left[\sum_{j=1}^{n} w_{k j}\left(w_{i j}-\cos \omega_{j} \frac{\left(x_{p i}-c_{i j}\right)}{\left\|x_{p}-c_{j}\right\|}\right) \operatorname{out}_{p j}^{(1)}\left(1-\operatorname{out}_{p j}^{(1)}\right)\right] \operatorname{out}_{p k}^{(2)}\left(1-\operatorname{out}_{p k}^{(2)}\right) .
$$

Using this formula, the network input that produces low sensitivity is determined. Considering that the feature that represents this input is insignificant, it is removed from the network (Hashem 1992; Neurosolutions 1999). The network is then trained and tested with the remaining features.

\section{Applications}

Sensitivity analysis for CSFNN was applied to various databases. These are thyroid disease, Iris plant pattern recognition and ionosphere databases.

\subsection{Thyroid disease diagnosis problem}

In the first application, thyroid disease has been classified by using CSFNN and sensitivity analysis applied to data. Thyroid data (Chatur \& Ghatol 2000; Hanford 2002) are measurements of the amounts of different hormones produced by the thyroid gland. This dataset contains three classes and 215 samples. These classes are assigned to the values that correspond to hyper-, hypo- and normal function of the thyroid gland. All samples have five features. These are as below.

1. T3-resin uptake test (a percentage).

2. Total serum thyroxin as measured by the isotopic displacement method.

3. Total serum triiodothyronine as measured by radioimmuno assay.

4. Basal thyroid-stimulating hormone (TSH) as measured by radioimmuno assay.

5. Maximal absolute difference of TSH value after injection of 200 micrograms of thyrotropin-releasing hormone as compared to the basal value.

The first class has 150 instances, the second has 35 instances and, finally, the third has 30 instances. A training set and a test set are formed using 215 samples. A three-fold crossvalidation method was used for this classification problem. In this cross-validation method, the data are divided into three sub-sets (A, B and C). The sets A and B are trained first and set $C$ is tested. In the second step, the sets $A$ and $C$ are trained and set B is tested. Finally, the sets $\mathrm{B}$ and $\mathrm{C}$ are trained and set $\mathrm{A}$ is tested. The results for each subset are given in table 1 .

The sensitivity analysis is then, applied to each subset. After applying the sensitivity analysis method, the input produces low sensitivity, which represents a feature, is found for each subset. Considering that the feature that represents this input is insignificant, it is removed from the network, and then, training and testing are repeated. The removed input and corresponding test results at each subset are given in table 2 . It should be noted that since the 
Table 1. Results for each subset of the thyroid database before applying sensitivity analysis.

\begin{tabular}{ccc}
\hline Subset & $\begin{array}{c}\text { Classification } \\
\text { accuracy(\%) }\end{array}$ & $\begin{array}{c}\text { Training } \\
\text { epoch }\end{array}$ \\
\hline A & 98.6 & 28 \\
B & 88.8 & 52 \\
C & 85.9 & 139 \\
\hline
\end{tabular}

Table 2. Results for each subset of thyroid database after applying sensitivity analysis.

\begin{tabular}{cccc}
\hline Subset & $\begin{array}{c}\text { Removed } \\
\text { input }\end{array}$ & $\begin{array}{c}\text { Classification } \\
\text { accuracy(\%) }\end{array}$ & $\begin{array}{c}\text { Training } \\
\text { epoch }\end{array}$ \\
\hline A & 4 & 98.6 & 25 \\
B & 3 & 88.8 & 99 \\
C & 4 & 84.5 & 41 \\
\hline
\end{tabular}

data distribution of each subset is different, the number of training epochs and the classification accuracy for subsets are also different. The fact that the different data distributions exist for subsets explains the increasing and decreasing at the epoch for subset ' $\mathrm{B}$ ' and subset ' $\mathrm{C}$ '. This fact also denotes that the removed input from the network changes for each subset.

Furthermore, another work has been done to show the merit of this method. Here, subset 'A' was chosen and each input has been removed from the network one by one without doing any analysis, and the network has been trained and tested for each cases. The results demonstrate that the fourth input has the lowest sensitivity as in figure 2. Here, $x$-axis indicates the number of removed input from the network. This agrees with the result of sensitivity analysis method for subset 'A' as shown in table 2.

\subsection{Iris plant classification problem}

Iris plant database, which is a well-known pattern recognition database, contains three classes of 50 instances each, where each class refers to a type of Iris plant (Fisher 1936; Blake 1988). A training set and a test set were formed using the 150 samples. The samples are represented by four features: sepal length, sepal width, petal length and petal width, all in centimetres. The training set contains 120 patterns, 40 from each pattern class. The remaining 30 patterns were used to test the training algorithms. A five-fold cross validation method has been used for this problem. The results obtained by removing the feature (petal width) with low sensitivity from the network are shown in figure 3. It is observed that all samples were classified correctly, but the training time was increased in this particular problem. This result is also compared with some other algorithms using five-fold cross validation method with four features: $k$-NN

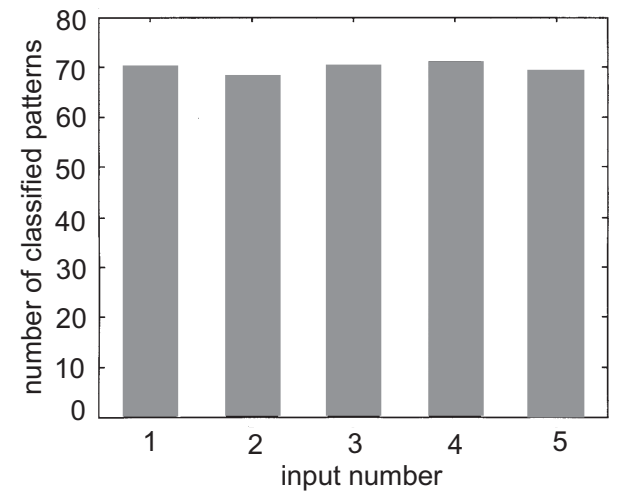

Figure 2. Variations in classification number with the removed inputs for ionosphere database. 


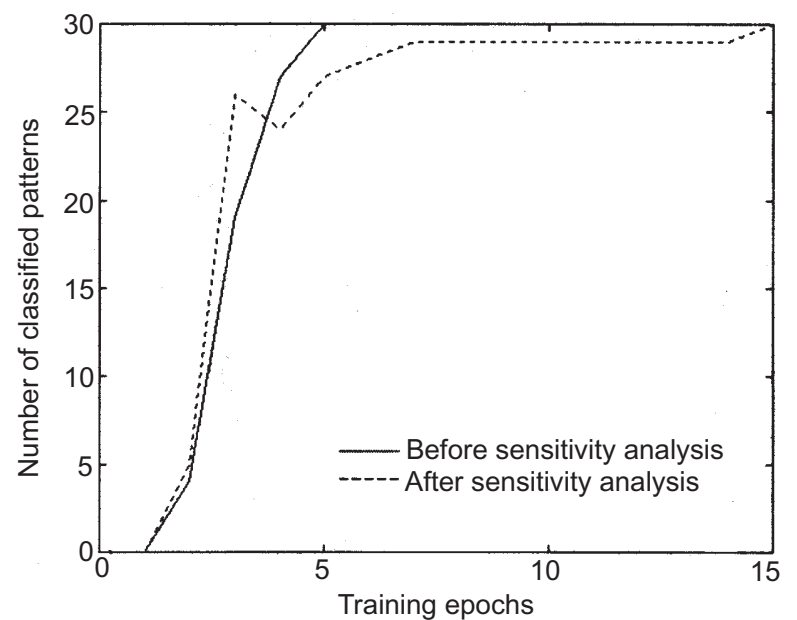

Figure 3. The results for Iris plant classification problem.

classifies the data with $95.34 \%$ accuracy and $k$-NNFP with 96.02\% accuracy (Ayan 1999). As it can be seen, even though fewer features have been used, the results obtained in this work are better than the others.

\subsection{Ionosphere database}

Finally, a larger database with 34 inputs was chosen to show the validity of method. This last database investigated was a radar data collected by a system in Goose Bay, Labrador. This system consists of a phased array of 16 high-frequency antennas with a total transmitted power on the order of 6.4 kilowatts (Sigillito et al, 1989). The targets were free electrons in the ionosphere. "Good" radar returns are those showing evidence of some type of structure in the ionosphere. "Bad" returns are those that do not allow their signals to pass through the ionosphere. Instances in this database are described by two attributes per pulse number, corresponding to the complex values returned by the autocorrelation function resulting from the received complex electromagnetic signal. The first 201 instances for training, which were carefully split into almost 50\% "good" and 50\% "bad", have been used for training and then the network has been tested with the remaining 150 test instances, consisting of 123 "good" and only 27 "bad" instances. According to test results, $97.3 \%$ classification accuracy was obtained, which is better than the reference work (Sigillito et al 1989) with an average of $96 \%$ accuracy on the test instances.

After sensitivity analysis was applied, the second input was found to be the insignificant input and it was removed from the network. Then, it was seen that the same accuracy has been obtained with the remaining 33 inputs. After that, the second and third inputs with the same sensitivity value have been removed from the network at the same time. In this case, the accuracy was $96.66 \%$. Later, the fourth and fifth less sensitive inputs have been also removed from the network one at a time. Classification accuracies of $97.3 \%$ and $96.66 \%$ were obtained respectively. Figure 4 shows the corresponding results. In this figure, the $x$-axis indicates how many inputs were removed from the network at the same time. Each input that removed from the network has different effect on the classification accuracy due to the different data distributions. Furthermore, these results show that removing more than one input with low sensitivity values from a large network does not greatly affect the classification accuracy. 


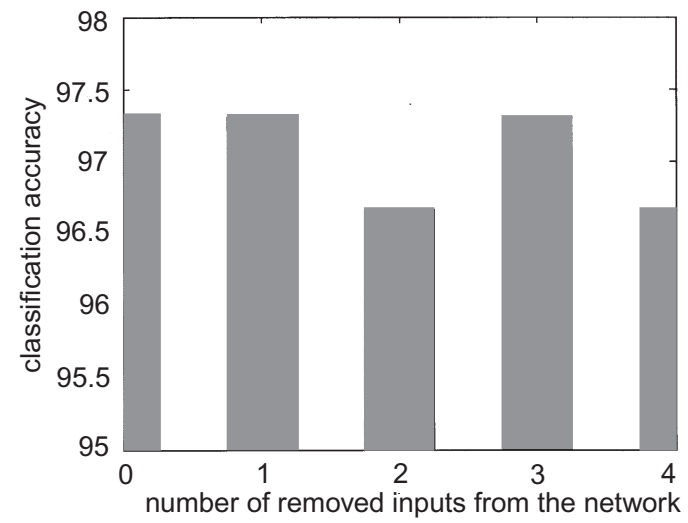

Figure 4. Variations in classification accuracy with the removed inputs for ionosphere database.

\section{Conclusions}

This paper has presented a sensitivity analysis method as an alternative to statistical approaches for aiming to reduce dimensionality and consequently connectivity in conic section function neural network. Thyroid disease diagnosis, Iris plant pattern recognition and ionosphere problems have been investigated. The inputs that produce low sensitivity values were considered insignificant and removed from the network for each database, and then the training and testing repeated. For all problems, comparative results obtained before and after sensitivity analysis method were given in terms of training epochs and classification accuracy. These results have been also compared with some reference work. Consequently, although the results were dependent on the problems, it was seen that the sensitivity analysis could be successfully used as a method to reduce the dimensionality and high connectivity requirement of networks. Particularly, ionosphere database results showed that removing more than one input with low sensitivity values from a large network does not greatly affect the accuracy. This may provide significant impetus for neural network circuit implementations in practice.

\section{List of symbols}
$p \quad$ number of patterns
$x_{p i} \quad$ input to neuron $i$ in the input layer for pattern $p$;
out $_{p k}^{(2)} \quad$ output of the $k$ th neuron in the output layer for pattern $p$;
out ${ }_{p j}^{(1)} \quad$ output of the $j$ th neuron in the hidden layer for pattern $p$;
$y_{p k}^{(2)} \quad$ activation of the $k$ th neuron in the output layer for pattern $p$;
$y_{p j}^{(1)} \quad$ activation of the $j$ th neuron in the hidden layer for pattern $p$;
$w_{i j} \quad$ weight between the $i$ th input and $j$ th hidden units;
$w_{j k} \quad$ weight between the $j$ th hidden and $k$ th output units;
$\theta \quad$ threshold value;
$\omega \quad$ opening angle of a cone. 


\section{References}

Acar A 1979 Sensitivity and tolerance analysis (Istanbul: Fatih)

Ayan N F 1999 Using information gain as feature weight. Turkish Symp. on Artificial Intelligence and Neural Networks, Istanbul, Turkey, pp 48-57

Basturk L, Gurgen F 1993 A study on the optimization and the sensitivity of multilayer perceptron. Proc. Cong. of Electrical Engineering, Trabzon, Turkey (in Turkish)

Blake C 1988 Iris plant database. Available at http://www.ics.uci.edu/pub/ml-repos/machine-learningdatabases/iris/iris.names

Broomhead D S, Lowe D 1988 Multivariable functional interpolating and adaptive networks. Complex Syst. 2: 321-355

Chatur P N, Ghatol A A 2000 Artificial neural networks for diagnosis of thyroid disease. (http://ieindia.org/publish/id0602.htm), ID0602 Interdisiplinary Journal

Delgado-Frias J G, Moore W R 1994 VLSI for neural networks and artificial intelligence (New York: Plenum)

Dorffner G 1994 Unified framework for MLPs and RBFNs: Introducing conic section function networks. Cybern. Syst. 25: 511-554

Elmasry M I (ed.) 1994 VLSI artificial neural networks engineering (Boston: Kluwer Academic)

Fakhr W, Kamel M, Elmasry M I 1994 Minimum complexity neural networks for classification. VLSI artificial neural networks engineering (ed.) M I Elmasry (Boston: Kluwer Academic)

Fisher R A 1936 The use of multiple measurements in taxonomic problems. Annu. Eugenics 7: 179188

Hanford 2002 Hanford thyroid disease study. Review of thyroid disease and approaches to diagnosis. Fred Hutchinson Cancer Research Center, Seattle, www.fhcrc.org/phs/htds/4nf-thdx.htm

Hashem S 1992 Sensitivity analysis for feedforward artificial neural networks with differentiable activation functions. Proc. of IJCNN'92 (Baltimore: IEEE) 1: 419-424

Haykin S 1994 Neural networks: a comprehensive foundation (New York: Macmillan)

NeuroSolutions 1999 Sensitivity analysis for NeuroSolutions. Available at http://www.nd.com/products/

Ozyilmaz L 2000 Developing of learning algorithm and investigating of the network performance and sensitivity for various problems in conic section function neural networks. $\mathrm{Ph} D$ thesis, Yildiz Technical University, Istanbul, Turkey

Poggio T, Girosi F 1990 Networks for approximation and learning. Proc. IEEE 78: 1481-1497

Ramacher U, Rückert U 1991 VLSI design of neural networks (Boston: Kluwer Academic)

Sigillito V G, Wing S P, Hutton L V, Baker K B 1989 Classification of radar returns from the ionosphere using neural networks. Johns Hopkins APL Tech. Dig. 10: 262-266, also at http://ftp.ics.uci.edu/pub/ml-repos/machine-learning-databases/ionosphere/

Yildirim T, Marsland J S 1997 A unified framework for connectionist models. Perspectives in neural computing series: Connectionist representations (London: Springer-Verlag) pp 26-39 